\title{
SkQ1 Ophthalmic Solution for Dry Eye Treatment: Results of a Phase 2 Safety and Efficacy Clinical Study in the Environment and During Challenge in the Controlled Adverse Environment Model
}

Anton Petrov $\cdot$ Natalia Perekhvatova $\cdot$ Maxim Skulachev •

Linda Stein · George Ousler

To view enhanced content go to www.advancesintherapy.com Received: July 2, 2015 / Published online: January 5, 2016

(C) The Author(s) 2016. This article is published with open access at Springerlink.com

\begin{abstract}
Introduction: This Phase 2 clinical trial assessed the efficacy and safety of the novel antioxidative, renewable compound SkQ1 for topical treatment of dry eye signs and symptoms.
\end{abstract}

Methods: In a single-center, randomized, double-masked, placebo-controlled, 29-day study, 91 subjects with mild to moderate dry eye instilled the study drug twice daily and recorded dry eye symptoms daily. Subjects were randomized 1:1:1 into one of three ophthalmic solution treatment groups: SkQ1 $1.55 \mu \mathrm{g} / \mathrm{mL}$, SkQ1 $0.155 \mu \mathrm{g} / \mathrm{mL}$, or $0.0 \mu \mathrm{g} / \mathrm{mL} \quad$ (placebo). Subjects were exposed to a controlled adverse environment chamber at 3 of the 4 study visits (Day -7, Day 1, and Day 29). Investigator assessments occurred at all study visits.

Electronic supplementary material The online version of this article (doi:10.1007/s12325-015-0274-5) contains supplementary material, which is available to authorized users.

A. Petrov · N. Perekhvatova · M. Skulachev

Mitotech, SA, Luxembourg City, Luxembourg

L. Stein · G. Ousler $(\bowtie)$

Ora, Inc., Andover, MA, USA

e-mail: gousler@oraclinical.com
Results: SkQ1 was safe and efficacious in treating dry eye signs and symptoms. Statistically significant improvements with SkQ1 compared to placebo occurred for the dry eye signs of corneal fluorescein staining and lissamine green staining in the central region and lid margin redness, and for the dry eye symptoms of ocular discomfort, dryness, and grittiness. In addition, SkQ1 demonstrated greater efficacy compared to placebo, although the differences were not statistically significant, for corneal fluorescein staining in other regions and/or time points (total staining score, central region, corneal sum score, and temporal region), lissamine green staining for the central and nasal regions, and blink rate scores. Conclusions: This Phase 2 study indicated that SkQ1 is safe and efficacious for the treatment of dry eye signs and symptoms and supported previous study results.

Trial registration: Clinicaltrials.gov identifier: NCT02121301.

Funding: Miotech S.A.

Keywords: Dry eye; SkQ1; Antioxidant; Controlled adverse environment; CAE; Mitochondria 


\section{INTRODUCTION}

The prevalence of dry eye syndrome worldwide ranges from $5 \%$ to $34 \%$ of the population [15], and in the US is estimated to affect over $4 \%$ of men and almost $8 \%$ of women 50 years of age or older [21, 22]. Dry eye syndrome, which increases with age, is a multifactorial disease of the tears and ocular surface; symptoms include discomfort, visual disturbance, and tear film instability, with the potential for exposure of and damage to the ocular surface [7]. Dry eye is accompanied by increased osmolarity of the tear film, which can lead to morphological changes in the cornea and conjunctiva. Dry eye also involves inflammation of the ocular surface, which can result in apoptotic cell death in the corneal epithelium [3, 37]. Current therapies for dry eye are only palliative, focusing on replacement of tears to reduce symptoms. Thus, there is a need for drugs that directly address the causes of dry eye.

Oxidative mechanisms are believed to play an important role in the pathogenesis of dry eye syndrome. SkQ1 is a novel small molecule developed to reduce oxidative stress in cell mitochondria, targeting and neutralizing mitochondrial reactive oxygen species (ROS). Mitochondria are one of the main sources of molecular oxygen consumption in the body $[12,29]$. Natural antioxidants are not capable of penetrating mitochondria and neutralizing the excessive amounts of free radicals generated by these organelles. After ROS-dependent oxidation in the mitochondria, SkQ1 is reduced by the respiratory chain, making it a renewable active compound [26]. The localization of SkQ1 in mitochondria and its ability to regenerate make it a potentially effective pharmacological agent for treating pathologies of the eye associated with oxidative stress and lipid and protein peroxidation in the inner membrane of the mitochondria, and for the prevention of the onset or progression of dry eye syndrome.

As a topical ophthalmic formulation for dry eye, SkQ1 has demonstrated statistically significant positive results in a Phase 2 clinical trial in the US for reduction of both signs and symptoms in subjects with mild to moderate dry eye syndrome, as discussed in this article. This study confirmed the results of a prior SkQ1 clinical study conducted in Russia and Ukraine [5]. An ophthalmic formulation of SkQ1, Visomitin, was approved in Russia in December 2011 and has since been marketed there as a prescription product. Russian studies with SkQ1 have involved 633 subjects; the US Phase 2 study enrolled 91 subjects.

\section{MATERIALS AND METHODS}

The Phase 2 US study was a randomized, double-masked, placebo-controlled, singlecenter, 29-day clinical trial. The study evaluated the safety and efficacy of SkQ1 ophthalmic solution in the environment and during challenge in the Controlled Adverse Environment $\left(\mathrm{CAE}^{\mathrm{SM}}\right)$ chamber (Ora, Inc., Andover, MA, USA). The CAE is a clinical model used in the investigation of therapeutic agents to exacerbate the signs and symptoms of dry eye in a controlled manner by regulating humidity, temperature, airflow, lighting, and visual tasking within the CAE chamber. The CAE allows for standardized measurements of dry eye signs and symptoms and reduces variability [18]. The study included several dry eye sign and symptom parameters, described below.

\section{Study Population}

The subject selection process ensured that no subjects with underlying factors that could 
affect the conduct of the study or compromise subject safety enrolled in the study. All 91 subjects enrolled had clinical signs of mild to moderate dry eye for at least 6 months prior to study entry. The mean age of subjects was 62 years [standard deviation (SD) 10.68]; 24 subjects were men and 67 were women. Key inclusion criteria at Visits 1 and 2 included a corneal fluorescein staining score of $\geq 2$ in at least one region pre-CAE, a total lissamine green conjunctival score of $\geq 2$ pre-CAE, a Schirmer's test score of $\leq 10$ and $\geq 1 \mathrm{~mm}$, a score of $\geq 2$ in at least one symptom pre-CAE, and a demonstrated response to the $\mathrm{CAE}$, based on Ora Calibra $^{\mathrm{TM}}$ scales (Ora, Inc., Andover, MA, USA) as discussed in this article.

All subjects had to have demonstrated a reproducible dry eye response to the CAE at Visits 1 and 2. Exclusion criteria included no recent history of ocular surgery/procedures, no clinically significant (CS) slit lamp findings, no ongoing ocular infection or inflammation, no Restasis $^{\circledR}$ (Allergan, Inc., Irvine, CA, USA) use within 30 days of Visit 1 (Day -7), no punctal plug use that was not stable within 30 days of Visit 1, no contact lens use within 7 days of Visit 1 , and no use of medications known to cause ocular drying within 30 days of Visit 1.

\section{Study Design}

All subjects who met the inclusion and exclusion criteria were randomized 1:1:1 into one of three ophthalmic solution treatment groups, at SkQ1 $1.55 \mu \mathrm{g} / \mathrm{mL}$, SkQ1 $0.155 \mu \mathrm{g} / \mathrm{mL}$, or SkQ1 $0.0 \mu \mathrm{g} / \mathrm{mL}$ (placebo). The placebo consisted of benzalkonium chloride, hypromellose, sodium chloride, sodium dihydrogen phosphate dihydrate, and sodium dihydrogen phosphate dodecahydrate, and was identical to the SkQ1 treatment drug formulation except that the active ingredient
SkQ1 was absent. Subjects self-administered one to two drops twice daily (BID) for 29 days in the morning and evening, including self-administration at two in-office visits, as described below. Subjects also recorded dry eye symptoms in a diary. Investigator assessments occurred at all study visits.

The study involved 4 visits over approximately 5 weeks. Visit 1 (Day -7) was the screening visit; subjects were assessed for eligibility, including response to a 90-min CAE exposure. The study included a 1-week placebo (SkQ1 vehicle) run-in period between Visit 1 and Visit 2 for subject selection prior to randomization. At Visit 2 (Day 1), baseline efficacy and safety measures were taken and baseline values established; subjects received a 90-min CAE exposure and their responses to the CAE were confirmed. Also at Visit 2, subjects were then randomized into the three treatment groups and self-administrated the assigned study drug. Subjects continued to self-administer the study drug BID for 4 weeks. Visit 3 (Day 15) was the 2-week follow-up visit with efficacy and safety measures taken, and included subject self-administration of the study drug; no CAE exposure occurred at Visit 3. At Visit 4 (Day 29), the final 90-min CAE exposure occurred, efficacy and safety assessment measures were taken, and subjects exited the study.

Most efficacy measures for dry eye signs (corneal fluorescein staining, lissamine green staining, ocular protection index, blink rate, tear film break-up time (TFBUT), conjunctival redness, and lid margin redness) were assessed at each study visit (both pre- and post-CAE at Visits 1, 2, and 4, and once at Visit 3 at which no CAE exposure occurred). Additional measures for dry eye signs included corneal sensitivity, which was assessed at all study visits pre-CAE only, and Schirmer's test, which was conducted at all study visits post-CAE only. 
Efficacy measures for dry eye symptoms included subject assessments of five symptoms (ocular discomfort, burning, dryness, grittiness, and stinging) assessed at all study visits (both pre- and post-CAE and at Visit 3); drop comfort assessed post-CAE at Visit 2 and Visit 3; and an ocular surface disease index (OSDI) questionnaire assessed pre-CAE at all visits.

Safety assessments for adverse events (AEs), visual acuity, and slit lamp biomicroscopy testing were conducted at all study visits; intraocular pressure (IOP), dilated fundoscopy, and pregnancy testing were conducted at Visits 1 and 4.

\section{Statistical Analysis}

SAS $^{\circledR}$ software Version 9.2 (SAS Institute, Middleton, MA, USA) was used for statistical analyses. Efficacy parameters were assessed at all study visits at all or some of the following time points: Visit 4 (Day 29)—Pre-CAE, Pre-CAE change from Baseline (Visit 2, Day 1), post-CAE, change from Visit 2 post-CAE, and change from pre- and post-CAE at Visit 4; and Visit 3 (Day 15) and Visit 3 change from Baseline. The unit of analysis for most efficacy endpoints was the worst eye of each subject, defined as the eye with the worse inferior corneal staining before CAE exposure at baseline (Visit 2, Day 1), or if inferior corneal staining was the same in both eyes, then the eye with the earliest onset of symptom reaction to the CAE at baseline. For other endpoints, the unit of analysis was the subject.

Two-sided two-sample $t$ tests were calculated for levels of significance. A level of statistical significance of 0.05 was used for differences between the SkQ1 treatments and placebo. Wilcoxon rank sum tests were used to compare each dose of SkQ1 ophthalmic solution and placebo and for count or rank-based data, including symptom scores at individual time points. Analysis of covariance models were used to include baseline values as covariates where appropriate. Mixed-effect models were used to analyze individual dry eye symptoms from the subject diary.

The primary and secondary efficacy analyses were performed on the intent-to-treat (ITT) population, which included all randomized subjects. Sensitivity analysis was performed on the per-protocol population, which excluded subjects with significant protocol deviations or who did not complete the study. Safety assessments were performed on the safety population, which included all subjects who received treatment from whom at least one safety measurement was obtained following the first dose of study drug.

\section{Study Procedures}

\section{Efficacy Measures}

The procedures used to assess efficacy measures are described below.

\section{Dry Eye Signs}

Corneal Fluorescein Staining Fluorescein staining of the cornea, commonly used to assess ocular surface damage, was assessed by the investigator using a slit lamp 3-5 min after instillation of $5 \mu \mathrm{L}$ of $2 \%$ preservative-free sodium fluorescein solution into the inferior conjunctival cul-de-sac of each eye. A Wratten \#12 yellow filter was used to enhance the ability to grade fluorescein staining. Staining was assessed in precisely defined regions (inferior, superior, central, temporal, and nasal) of the ocular surface using the Ora Calibra ${ }^{\mathrm{TM}}$ Corneal and Conjunctival Staining Scale and the National Eye Institute (NEI)/Industry Workshop Scale (NEI scale) [13]. The Ora Calibra 5-point scale ranges from 0 to 4 points 
( $0=$ none to $4=$ confluent $)$ [23]. The 4 -point NEI scale ranges from $0=$ no staining present to $3=$ severe staining.

Lissamine Green Staining Lissamine green staining, another measure of ocular surface damage, was assessed by the investigator using a slit lamp approximately $30 \mathrm{~s}$ after instillation of $10 \mu \mathrm{L}$ of lissamine green solution into the inferior conjunctival cul-de-sac. The Ora Calibra Corneal and Conjunctival Staining Scale and the NEI scale (both scales described above) were used for assessment. The NEI grid divides the conjunctiva into six regions: far temporal, temporal-superior, temporal-inferior, nasalsuperior, nasal-inferior, and far nasal; the intensity of staining in these regions was scored by the investigator using the NEI 0-3 scoring system.

Ocular Protection Index (OPI 2.0) An automated methodology was used to simultaneously measure TFBUT (described below) and Inter Blink Interval (IBI, the average time between blinks), as well as the area of tear film break up (percent of cornea exposed). Mean breakup area divided by IBI provides the OPI 2.0 score, reflecting a percentage of the area of tear film break up. Following instillation of fluorescein solution into the inferior conjunctival cul-de-sac, subjects' tear films were recorded using a camera system for one minute while the subjects watched television. A 15 frame-per-second video of the fluorescent eye under visual task was taken and a computer program analyzed the area of cornea with broken tear film on a frame-by-frame basis. IBI and OPI were calculated and produced as assessments of the level of drying of the ocular surface.

Blink Rate Blinks per minute were measured over a three-minute period, with a lower blink rate after treatment and CAE exposure associated with improvements in dry eye. A subject's blink rate (blinks per minute) was non-invasively assessed using automated controls and measured over a period of up to 10 min using a digital micro-camera system. The camera tracked the position of the upper eyelid over time and counted the number of times the upper eyelid moved out of its resting position. The blink rate was taken while the subject focused on a visual task. The camera was mounted onto a headset that fit any subject and used ambient illumination.

TFBUT The TFBUT test was performed in accordance with standard procedures. For each eye, two measurements were taken and averaged unless the two measurements were $>2 \mathrm{~s}$ apart and were each $<10 \mathrm{~s}$, in which case, a third measurement was taken and the two closest of the three were averaged and used for analysis. If the difference between the two sequential pairs was the same (e.g., 3, 6, 9 s), then the median of the three readings was used for analysis. Less time indicated a faster break-up of the tear film, which in turn indicates an unstable tear film characteristic of evaporative dry eye.

Corneal Sensitivity Corneal sensitivity was measured with a Cochet-Bonnet aesthesiometer, using vertical nylon threads of different lengths $(\mathrm{mm})$ that touch the corneal surface. A lower value indicates less corneal sensitivity. The length at which the subject reported stimulus was recorded as the corneal sensitivity.

Unanesthetized Schirmer's Test A sterile Schirmer's strip was placed in the lower temporal lid margin for $5 \mathrm{~min}$, at which point the length of moistened strip was recorded as an 
indication of tear production. A normal Schirmer's test results in $\geq 15 \mathrm{~mm}$ wetting of the paper after $5 \mathrm{~min}$; lower values indicate more severe lack of tear production (a sign of dry eye syndrome).

Conjunctival Redness Conjunctival redness was assessed by the investigator using the 5-point (0-4) Ora Calibra Conjunctival Redness Scale $(0=$ none, and $4=$ most severe $)$.

Lid Margin Redness Lid margin redness was assessed by the investigator using the Ora Calibra 4-point (0-3) Lid Margin Redness Scale $(0=$ none, and $3=$ most severe $)$.

\section{Dry Eye Symptoms}

Ocular Discomfort Ocular discomfort scores were subjectively graded by the subjects according to the 5-point Ora Calibra Ocular Discomfort Scale $(0-4$, with $0=$ no discomfort and $4=$ constant discomfort) [23]. Each eye was rated separately. Subjects assessed ocular discomfort at time 0 and every $5 \mathrm{~min}$ thereafter during the 90-min CAE exposures. Subjects also assessed ocular discomfort at Visits 1,2 , and 4, before and after the CAE exposure, and also at Visit 3 when no CAE exposure occurred.

Dry Eye Symptoms and Ocular Discomfort Other dry eye symptoms as well as ocular discomfort were assessed for both eyes of each subject at all scheduled visits, pre-CAE and post-CAE at Visits 1, 2, and 4 and once at Visit 3 (Day 15) when no CAE was performed. The symptoms assessed by subjects were: overall ocular discomfort, burning, dryness, grittiness, and stinging, according to the 6-point (0-5) Ora Calibra $^{\mathrm{TM}}$ Ocular Discomfort and 4-Symptom Questionnaire, in which $0=$ none and $5=$ most.
Drop Comfort Drop comfort was assessed for each eye by the subject immediately following initial dosing with the study drug and at 1 and 2 min following dosing using the Ora Calibra Drop Comfort Scale, where $0=$ very comfortable and $10=$ very uncomfortable. Subjects also assessed drop comfort at $3 \mathrm{~min}$ following initial dosing using the Ora Calibra Drop Comfort Questionnaire by choosing 3 descriptor words from a list of 12 words (i.e., burning, cool, sticky) or inserting their own descriptor as part of the questionnaire.

Ocular Surface Disease An OSDI Questionnaire was used to evaluate the impact of a subject's dry eye disease on vision-related functioning. A negative change from baseline indicated an improvement in vision-related functioning. Study staff asked the subject a series of 12 questions related to the frequency of occurrence of dry eye symptoms over the previous week. Subjects rated each eye using a 5 -point scale where $0=$ none of the time and $4=$ all of the time.

\section{Safety Measures}

The procedures used to assess safety measures are described below.

\section{AEs}

All AEs regardless of relationship to the test article were monitored, reported, and recorded throughout the study.

\section{Visual Acuity}

Best corrected visual acuity was assessed for both eyes at all study visits (pre-CAE at visits in which CAE occurred) using an ETDRS chart. Study staff indicated on the case report form whether VA was measured with or without 
correction and if pin-hole was used. Number of letters read correctly was converted to logarithm of the minimum angle of resolution (logMAR) scores.

\section{Slit Lamp Biomicroscopy}

Slit lamp biomicroscopy examinations were conducted on both eyes at all study visits (pre-CAE and post-CAE at visits in which CAE occurred). The slit lamp findings included examinations of cornea, conjunctiva, anterior chamber, iris, lens, and lid. Each parameter was graded as normal or abnormal. Abnormal findings were further classified as CS or not CS (NCS).

\section{Dilated Fundoscopy}

Dilated fundoscopy examinations were conducted on both eyes at Visit 1 (Day -7) and Visit 4 (Day 29) post-CAE. The fundus pathology findings included examinations of the vitreous, retina, macula, choroid, and optic nerve, and were recorded as normal, abnormal non-clinically significant (NCS), or abnormal clinically significant (CS).

\section{IOP}

IOP was measured by contact tonometry on both eyes at Visits 1 and 4 (post-CAE).

\section{Dose Selection}

In a scopolamine and dry eye CAE mouse model study (70 mice) with SkQ1 ophthalmic solution $0.155 \mu \mathrm{g} / \mathrm{mL}$ as topical treatment $(3 \mu \mathrm{L})$ on days 1-12, SkQ1 statistically significantly reduced corneal staining for the once daily (QD) and BID doses compared to vehicle alone, with QD administration scores lower than vehicle at Day $4(p<0.01)$, and BID administration lower than vehicle at days $4 \quad(p<0.01)$ and 12 $(p<0.05)$. These results suggested that SkQ1 ophthalmic solution $0.155 \mu \mathrm{g} / \mathrm{mL}$ had a rapid onset of action as well as a long duration of action, that BID dosing was optimal, and that clinical testing of SkQ1 as a dry eye treatment was warranted [35].

Animal study data on SkQ1 lead to the selection of two test concentrations in the Phase 2 US study: the $0.155 \mu \mathrm{g} / \mathrm{mL}$ concentration used in preclinical studies, and a higher $1.55 \mu \mathrm{g} / \mathrm{mL}$ concentration. In a local tolerance study (acute eye irritation in rabbits), a single dose of $155 \mathrm{ng} /$ $\mathrm{mL}(0.015 \mu \mathrm{g} / \mathrm{eye})$ showed no ocular irritation. A repeat dose study (28-day ocular toxicity with 14-day recovery period in rabbits) tested three concentrations of the SkQ1 ophthalmic solution and demonstrated that $0.15 \mu \mathrm{g} /$ eye three times daily (TID) $(0.45 \mu \mathrm{g} /$ eye/day) showed no ocular irritation; $1.5 \mu \mathrm{g} / \mathrm{mL}$ TID (4.5 $\mu \mathrm{g} /$ eye/day) showed no-observedadverse-effect-level (NOAEL) for ocular toxicity; and $15 \mu \mathrm{g} /$ eye TID (45 $\mu \mathrm{g} /$ eye/day) showed mild ocular irritation/NOAEL for systemic toxicity.

With a 0.1-mL dose at the highest concentration $\quad(0.1 \mathrm{~mL} \times 1.55 \mu \mathrm{g} / \mathrm{mL} \quad$ BID $=$ $0.31 \mu \mathrm{g} /$ eye/day), the amount of SkQ1 instilled falls within a safety margin of 10 for systemic exposure observed at the NOAEL for ocular toxicity, $4.5 \mu \mathrm{g} / \mathrm{eye} / \mathrm{day}$. The lower concentration $\quad(0.1 \mathrm{~mL} \times 0.155 \mu \mathrm{g} / \mathrm{mL} \quad \mathrm{BID}=$ $0.031 \mu \mathrm{g} /$ eye/day) falls well within the same safety margin. Data indicated that SkQ1 ophthalmic solution (0.155 and $1.55 \mu \mathrm{g} / \mathrm{mL})$ could be expected to be safe and well tolerated in monitored human subjects studied at these concentrations $(1.55 \mu \mathrm{g} / \mathrm{mL} \quad \mathrm{BID}=3.1 \mu \mathrm{g} / \mathrm{eye} /$ day and $0.155 \mu \mathrm{g} / \mathrm{mL} \quad \mathrm{BID}=0.31 \mu \mathrm{g} /$ eye $/$ day) . Thus, the preclinical studies supported dosing at the higher concentration of $1.55 \mu \mathrm{g}$ SkQ1/mL, with no evidence of systemic exposure at either $1.55 \mu \mathrm{g} / \mathrm{mL}$ or $0.155 \mu \mathrm{g} / \mathrm{mL}$ with BID dosing. 


\section{Compliance with Ethics Guidelines}

The study was Institutional review board-approved (Alpha IRB) and registered (Clinicaltrials.gov identifier: NCT02121301).

All procedures followed were in accordance with the ethical standards of the responsible committee on human experimentation (institutional and national) and with the Helsinki Declaration of 1964, as revised in 2013. Informed consent was obtained from all patients at the beginning of the study.

\section{RESULTS}

Of the 91 randomized subjects, 87 subjects completed the study, and four discontinued, one for a protocol deviation and 3 by subject choice.

Results for several efficacy measures in this Phase 2 study indicated that SkQ1 was efficacious in treating dry eye signs and symptoms, even though findings did not meet the primary efficacy endpoints for this study of statistically significant treatment differences between SkQ1 and placebo in mean score pre-CAE for corneal fluorescein staining in the inferior region at Visit 4 (Day 29), and in mean score for worst dry eye symptom over the 7 days preceding Visit 4.

For dry eye signs, statistically significant results demonstrating SkQ1 efficacy occurred for corneal fluorescein staining, lissamine green conjunctival staining, and lid margin redness. For dry eye symptoms, statistically significant results occurred that indicated less ocular discomfort, dryness, and grittiness for SkQ1-treated subjects compared to subjects treated with placebo. Additional dry eye sign measurements, although not statistically significant, also indicated superior SkQ1 efficacy compared to placebo, and included corneal fluorescein staining for all other corneal regions at some time points, lissamine green staining for the central and nasal conjunctival regions, and blink rate.

Regarding safety, both doses of SkQ1 were safe. No serious AEs occurred, rates of AEs were similar between the SkQ1 treatment groups and the placebo group, and no subjects discontinued the study because of AEs. No CS safety issues were identified. Subjects reported good comfort and tolerability with SkQ1.

Results for each of the efficacy and safety parameters are discussed below.

\section{Corneal Fluorescein Staining}

As shown in Table 1, both concentrations of SkQ1 decreased corneal fluorescein staining more than placebo treatment at Visit 4 (Day 29) for change from pre- to post-CAE in 7 of 8 corneal regions and for post-CAE in 3 of 8 regions (5 of 8 regions for $1.55 \mu \mathrm{g} / \mathrm{mL}$ SkQ1), indicating a reduction (improvement) in this dry eye sign. Using the Ora Calibra scale, $1.55 \mu \mathrm{g} / \mathrm{mL}$ SkQ1 was statistically significantly more effective than placebo $(p=0.0215)$ in reducing central corneal fluorescein staining at Visit 4 (Day 29) for mean change from pre- to post-CAE.

Using the NEI scale, mean central fluorescein staining scores for this same time point indicated statistical significance for the $1.55 \mu \mathrm{g} / \mathrm{mL}$ SkQ1 $(p=0.0291)$ for reduction of central corneal staining. In addition, for several other corneal regions/scores, SkQ1 $0.155 \mu \mathrm{g} / \mathrm{mL}$ demonstrated superior efficacy compared to placebo, although the differences were not statistically significant, including total staining, the central region, and the corneal sum score (sum of scores from the inferior, 
Table 1 Corneal fluorescein staining scores post-CAE ${ }^{S M}$

\begin{tabular}{|c|c|c|c|c|}
\hline \multirow[t]{2}{*}{ Time point } & \multirow{2}{*}{ 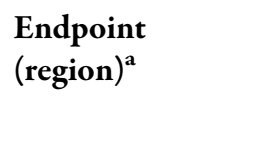 } & \multicolumn{3}{|c|}{ Mean $(S D)(p \text { value })^{\mathbf{b}}$} \\
\hline & & $\begin{array}{l}1.55 \mu \mathrm{g} / \mathrm{mL} \\
(N=30)\end{array}$ & $\begin{array}{l}0.155 \mu \mathrm{g} / \mathrm{mL} \\
(N=30)\end{array}$ & $\begin{array}{l}\text { Placebo } \\
(N=31)\end{array}$ \\
\hline Visit 4 & Total score & $10.92(2.918)$ & $11.12(2.912)$ & $10.85(3.217)$ \\
\hline \multirow[t]{12}{*}{ Post-CAE (Day 29) } & Corneal sum & $6.80(2.136)$ & $6.98(1.882)$ & $6.68(2.246)$ \\
\hline & Conjunctival sum & $4.12(1.092)^{c}$ & $4.13(1.438)$ & $4.17(1.295)$ \\
\hline & & $(p=0.8871)$ & $(p=0.9252)$ & \\
\hline & Inferior & $2.86(0.896)$ & $2.97(0.880)$ & $2.90(0.894)$ \\
\hline & & $(p=0.8695)$ & & \\
\hline & Superior & $2.44(0.754)$ & $2.52(0.701)$ & $2.25(0.751)$ \\
\hline & Central & $1.50(0.979)$ & $1.50(0.861)$ & $1.53(1.008)$ \\
\hline & & $(p=0.9020)$ & $(p=0.8909)$ & \\
\hline & Temporal & $1.94(0.618)$ & $1.97(0.718)$ & $1.97(0.669)$ \\
\hline & & $(p=0.8794)$ & & \\
\hline & Nasal & $2.18(0.593)$ & $2.17(0.781)$ & $2.20(0.750)$ \\
\hline & & $(p=0.9143)$ & $(p=0.8666)$ & \\
\hline Visit 4 & Total score & $-0.70(2.160)$ & $-1.23(2.690)$ & $-0.95(2.578)$ \\
\hline \multirow[t]{9}{*}{ Change from Visit 2 post-CAE } & Corneal sum & $-0.32(1.513)$ & $-0.82(1.709)$ & $-0.68(1.653)$ \\
\hline & & & $(p=0.7599)$ & \\
\hline & Conjunctival sum & $-0.38(1.348)$ & $-0.42(1.352)$ & $-0.27(1.172)$ \\
\hline & Inferior & $-0.20(0.707)$ & $-0.27(0.716)$ & $-0.25(0.679)$ \\
\hline & & $(p=0.7906)$ & & \\
\hline & Superior & $-0.02(0.530)$ & $-0.20(0.826)$ & $-0.27(0.751)$ \\
\hline & Central & $-0.10(0.816)$ & $-0.35(0.658)$ & $-0.17(0.699)$ \\
\hline & Temporal & $-0.12(0.794)$ & $-0.18(0.725)$ & $-0.07(0.691)$ \\
\hline & Nasal & $-0.26(0.818)$ & $-0.23(0.785)$ & $-0.20(0.690)$ \\
\hline Visit 4 & Total score & $1.36(2.452)$ & $1.05(2.131)$ & $2.20(2.973)$ \\
\hline \multirow{5}{*}{$\begin{array}{l}\text { Change from pre- to post- CAE } \\
\text { (Day 29) }\end{array}$} & & $(p=0.2643)$ & $(p=0.0904)$ & \\
\hline & Corneal sum & $1.06(1.764)$ & $0.90(1.694)$ & $1.63(2.105)$ \\
\hline & & $(p=0.2844)$ & $(p=0.1425)$ & \\
\hline & Conjunctival sum & 0.30 (1.099) & $0.15(0.882)$ & $0.57(1.158)$ \\
\hline & & $(p=0.3881)$ & $(p=0.1223)$ & \\
\hline
\end{tabular}


Table 1 continued

\begin{tabular}{|c|c|c|c|c|}
\hline \multirow[t]{2}{*}{ Time point } & \multirow{2}{*}{$\begin{array}{l}\text { Endpoint } \\
{\text { (region })^{\mathrm{a}}}^{\text {E }}\end{array}$} & \multicolumn{3}{|c|}{ Mean $(\mathrm{SD})(p \text { value })^{\mathbf{b}}$} \\
\hline & & $\begin{array}{l}.55 \mu \mathrm{g} / \mathrm{mL} \\
(N=30)\end{array}$ & $\begin{array}{l}0.155 \mu \mathrm{g} / \mathrm{mL} \\
(N=30)\end{array}$ & $\begin{array}{l}\text { Placebo } \\
(N=31)\end{array}$ \\
\hline & \multirow[t]{2}{*}{ Inferior } & \multirow[t]{2}{*}{$0.80(0.901)$} & $0.57(0.666)$ & \multirow[t]{2}{*}{$0.78(0.926)$} \\
\hline & & & $(p=0.3023)$ & \\
\hline & \multirow[t]{2}{*}{ Superior } & $0.34(0.703)$ & $0.23(0.817)$ & \multirow[t]{2}{*}{$0.35(0.892)$} \\
\hline & & $(p=0.9639)$ & $(p=0.5994)$ & \\
\hline & \multirow[t]{2}{*}{ Central } & $-0.08(0.965)$ & $0.10(0.845)$ & \multirow[t]{2}{*}{$0.50(0.851)$} \\
\hline & & $(p=0.0215)$ & $(p=0.0728)$ & \\
\hline & \multirow[t]{2}{*}{ Temporal } & $0.12(0.711)$ & $0.03(0.507)$ & \multirow[t]{2}{*}{$0.28(0.762)$} \\
\hline & & $(p=0.4184)$ & $(p=0.1402)$ & \\
\hline & \multirow[t]{2}{*}{ Nasal } & $0.18(0.497)$ & $0.12(0.568)$ & \multirow[t]{2}{*}{$0.28(0.583)$} \\
\hline & & $(p=0.4874)$ & $(p=0.2663)$ & \\
\hline
\end{tabular}

$C A E$ controlled adverse environment, $S D$ standard deviation

a Scales: Each region is assessed on a 0-4 scale with a ' 4 ' indicating the most staining; half (0.5) units may be used Corneal sum: sum of the inferior, superior, and central regions resulting in a corneal sum reported on a $0-12$ scale

Conjunctival sum: sum of the temporal and nasal regions resulting in a conjunctival sum reported on a $0-8$ scale

Total sum: sum of all five regions: inferior, superior, central, temporal, and nasal, resulting in a total sum reported on a $0-20$ scale

b 2 -sided $p$ value calculated using a two-sample $t$ test

c Results indicating numerical superiority of SkQ1 over placebo and associated $p$ values are shown in bold type. CAE was not conducted at Visit 3

superior, and central regions) (Visit 4 change from pre- to post-CAE); and the temporal region (Visit 3 change from baseline).

Figure 1 shows the differences in central corneal fluorescein staining scores in the ITT population comparing pre- and post-CAE at Day 1, and Fig. 2 shows the same parameters at Day 29.

\section{Lissamine Green Staining}

Using the Ora Calibra scale, the $0.155 \mu \mathrm{g} / \mathrm{mL}$ SkQ1 concentration was statistically significantly more effective than placebo $(p=0.0458)$ in reducing lissamine green staining for the central region at Visit 4 mean change from pre- to post-CAE, with scores of

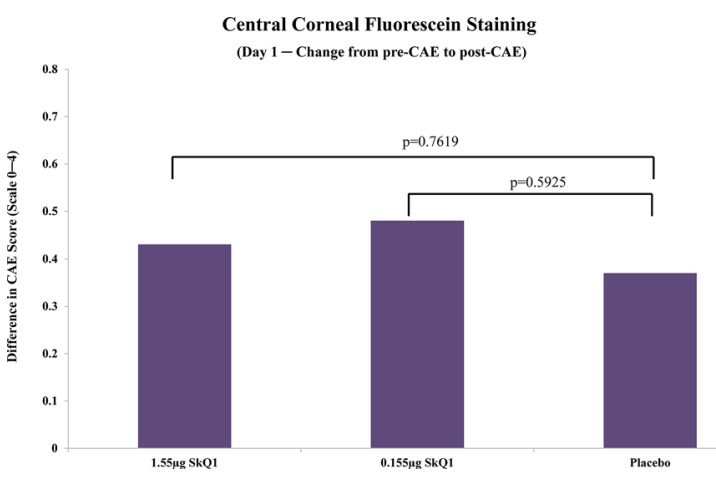

Fig. 1 Differences in central corneal fluorescein staining scores in the ITT population when compared pre- and post-CAE ${ }^{S M}$ at Day 1. Lower corneal staining scores indicate less dry eye. CAE controlled adverse environment, ITT intent-to-treat

-0.12 (SD 0.313) for the 0.155 SkQ1 group and 0.00 (SD 0.000) for the placebo group (lower scores reflect less dry eye). In addition, for other 


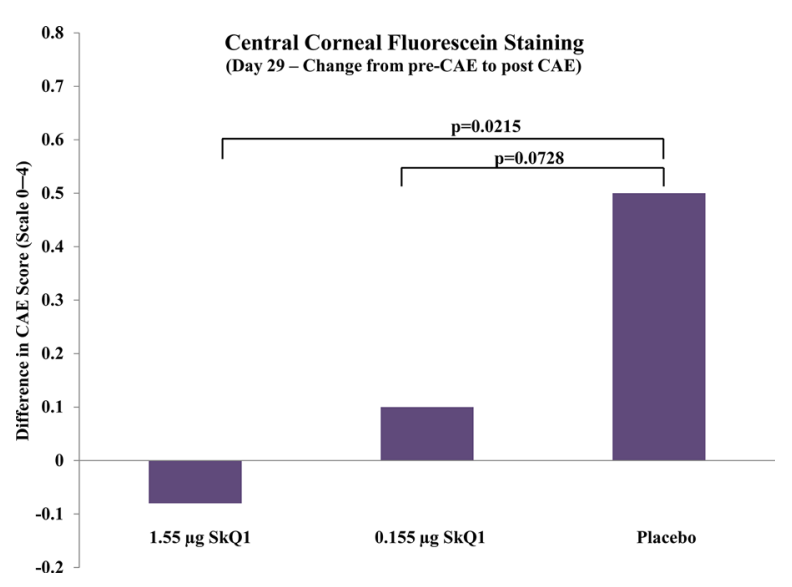

Fig. 2 Differences in central corneal fluorescein staining scores in the ITT population when compared pre- and post-CAE $^{\mathrm{SM}}$ at Day 29. Lower corneal staining scores indicate less dry eye. The SkQ1 $1.55 \mu \mathrm{g} / \mathrm{mL}$ dose had statistically significant reduction in central corneal fluorescein staining compared to placebo. There was a trend towards statistically significant lower staining for the SkQ1 $0.155 \mu \mathrm{g} / \mathrm{mL}$ dose compared to placebo. CAE controlled adverse environment, ITT intent-to-treat

time points, SkQ1 demonstrated superior efficacy compared to placebo in reducing lissamine green staining, although the differences were not statistically significant, including the central region (Visit 4 change from pre-CAE baseline, $1.55 \mu \mathrm{g} / \mathrm{mL}$ SkQ1) and nasal region (Visit 4 change from post-CAE baseline, both SkQ1 concentrations).

\section{Lid Margin Redness}

There were statistically significant improvements (decreases) in lid margin redness scores at several time points for both SkQ1 concentrations compared to placebo. This occurred for the $1.55 \mu \mathrm{g} / \mathrm{mL} \quad$ SkQ1 concentration at Visit 4 for: post-CAE $(p=0.0039)$, post-CAE change from Visit 2 post-CAE baseline $(p=0.0098)$, and pre- to post-CAE $(p=0.0224)$. Statistically significant efficacy also occurred for the $0.155 \mu \mathrm{g} / \mathrm{mL}$ SkQ1 concentration compared to placebo at Visit 4 for pre-CAE $(p=0.0319)$, post-CAE $(p=0.0028)$, and post-CAE change from Visit 2 post-CAE baseline $(p=0.0451)$. At Visit 3, SkQ1 $0.155 \mu \mathrm{g} / \mathrm{mL}$ showed greater efficacy in reducing lid margin redness compared to placebo, although the differences were not statistically significant.

\section{OPI}

There were no statistically significant differences in OPI values between either of the SkQ1 concentrations and placebo at Visits 3 and 4.

\section{Blink Rate}

Blink rate scores for the $1.55 \mu \mathrm{g} / \mathrm{mL}$ SkQ1 concentration demonstrated greater SkQ1 efficacy (i.e., a decrease in scores) compared to placebo at Visit 4 for change from Visit 2 post-CAE baseline, and at Visit 4 change from pre- to post-CAE. There were no important differences in blink rate between the $0.155 \mu \mathrm{g} /$ $\mathrm{mL}$ SkQ1 concentration and placebo throughout the study.

The remaining efficacy measures for signs of dry eye (TFBUT, conjunctival redness, corneal sensitivity, and Schirmer's test) either did not show statistically significant differences between the SkQ1 groups and the placebo group or showed significance for the placebo.

\section{Dry Eye Symptoms}

Table 2 reflects the results of several dry eye symptom analyses. Dry eye symptom scores for ocular discomfort for SkQ1 (using the Ora Calibra Ocular Discomfort and 4-Symptom Questionnaire) showed statistically significant improvement for SkQ1 $0.155 \mu \mathrm{g} / \mathrm{mL}$ relative to placebo $(p=0.0137)$ at Visit 4 (Day 29, change 


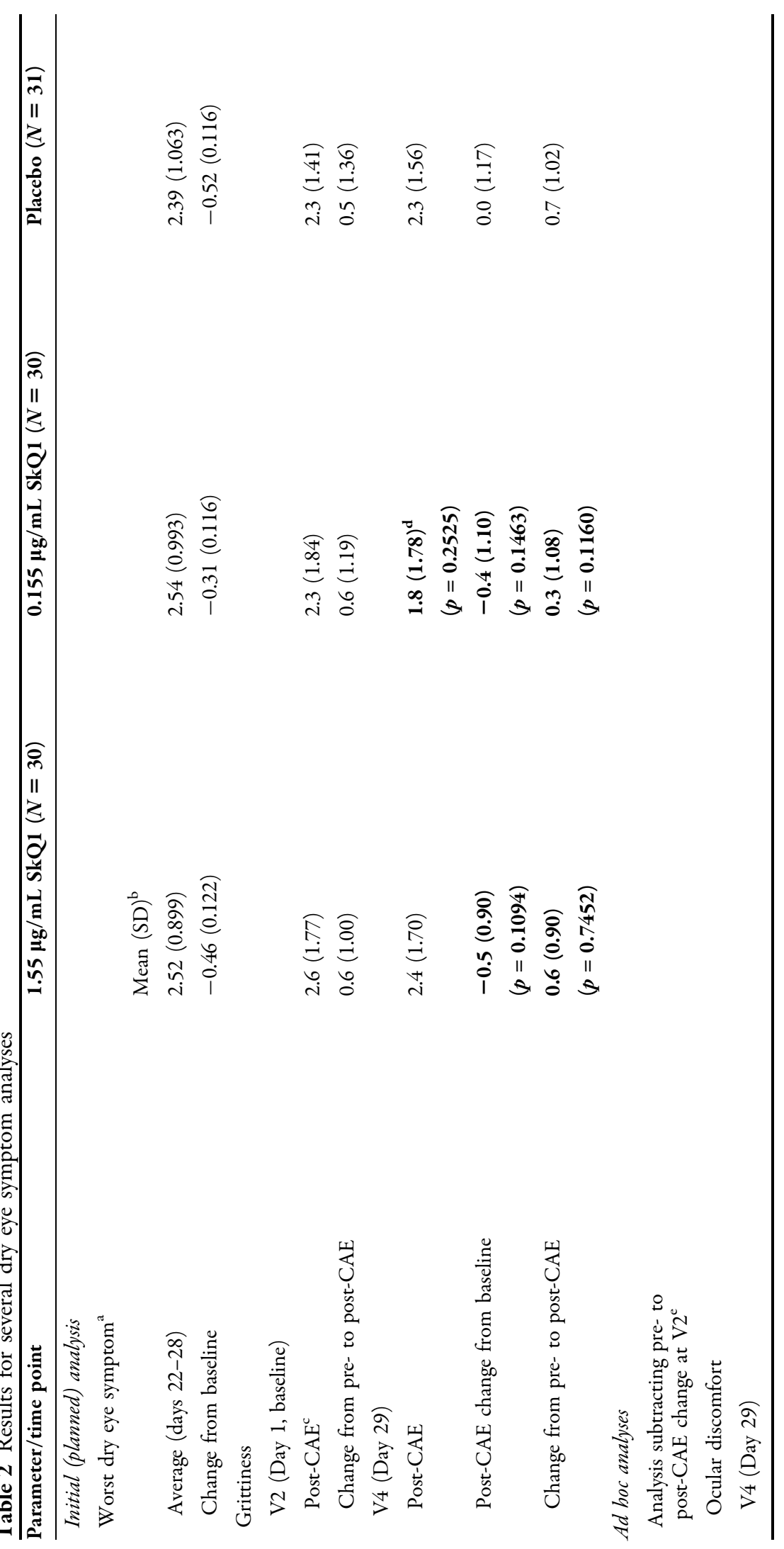




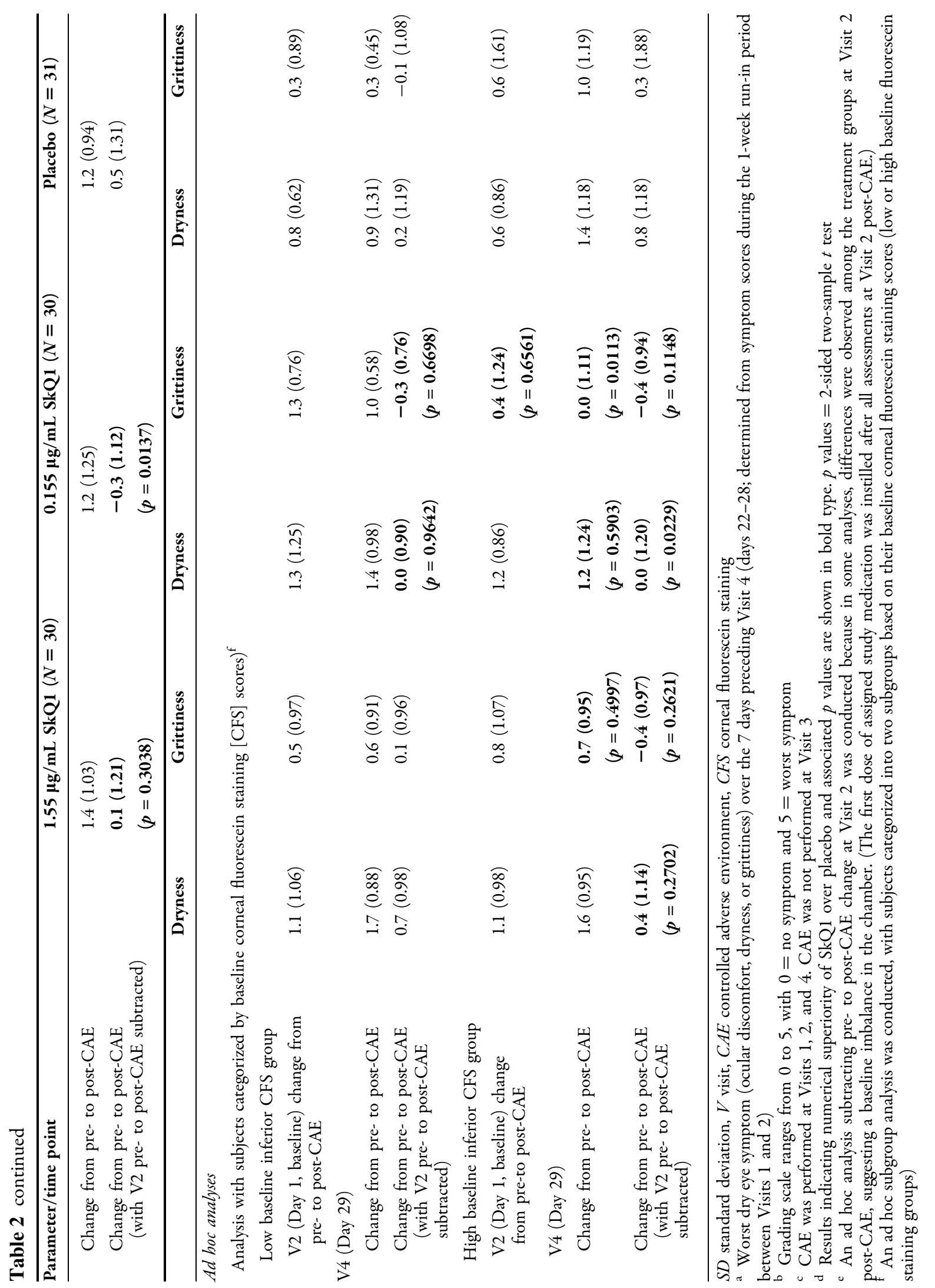




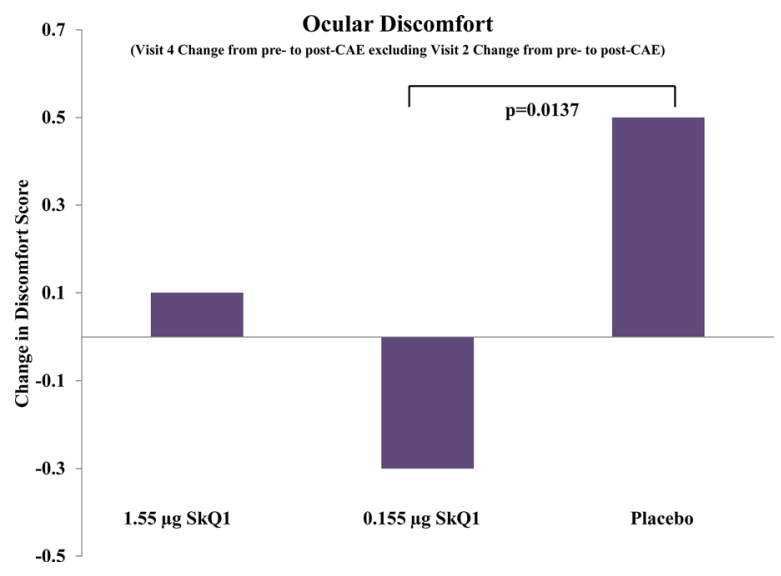

Fig. 3 Ocular discomfort scores for SkQ1compared to placebo. Lower ocular discomfort scores indicate less discomfort. Ocular discomfort in the ITT population at Visit 4 (Day 29) pre- to post-CAE ${ }^{\text {SM }}$ (with Visit 2 baseline change from pre- to post-CAE subtracted) was statistically significantly reduced in the SkQ1 $0.155 \mu \mathrm{g} / \mathrm{mL}$ treatment group compared to the placebo group. Ocular discomfort was also notably lower in the SkQ1 $1.55 \mu \mathrm{g} / \mathrm{mL}$ treatment group compared to placebo. CAE controlled adverse environment, ITT intent-to-treat

from pre- to post-CAE) in an ad hoc analysis subtracting pre- to post-CAE change at Visit 2 . This analysis was performed because in some analyses, differences were observed among the treatment groups at Visit 2 post-CAE, suggesting a baseline imbalance in the chamber. (The first dose of assigned study medication was instilled after all assessments at Visit 2 post-CAE.) Figure 3 shows the differences in ocular discomfort scores at Visit 4 (Day 29) compared to Baseline (Visit 2) in this analysis.

When subjects were categorized into subgroups according to their baseline corneal fluorescein staining scores (Visit 2, pre-CAE), dryness was statistically significantly lower $(p=0.0229) \quad$ in the SkQ1 $0.155 \mu \mathrm{g} / \mathrm{mL}$ high-baseline subgroup compared to the placebo group. Generally, the treatment effect of SkQ1 on symptoms was enhanced when evaluating subjects with higher baseline levels of staining.
In addition, in the general, planned analysis, grittiness scores at Visit 4 for change from baseline post-CAE and change from pre- to post-CAE demonstrated greater efficacy at both SkQ1 concentrations compared to placebo, and in the ad hoc subgroup analysis, at Visit 4 the grittiness score for change from pre- to post-CAE in the $0.155 \mu \mathrm{g} / \mathrm{mL}$ treatment group for subjects with High Baseline Inferior Corneal Staining was statistically significant $(p=0.0113)$ compared to placebo.

Using the Ora Calibra Drop Comfort Scale, no statistically significant treatment differences were found in comfort levels for either concentration of SkQ1 compared to placebo at any of the time points evaluated (Visit 2 and Visit 3). Subjects in all three treatment arms generally rated the drops as comfortable. Using the Ora Calibra Drop Comfort Questionnaire, the most commonly chosen adjectives for all three treatment groups were positive, and included cool, comfortable, soothing, and refreshing. In addition, using the OSDI, subject responses regarding poor vision indicated fewer dry eye symptoms with SkQ1 use compared to placebo (at Visit 4, and Visit 4 change from baseline).

\section{Safety Results}

\section{AEs}

Both doses of SkQ1 ophthalmic solution (1.55 and $0.155 \mu \mathrm{g} / \mathrm{mL}$ ) were safe and well tolerated as administered (BID) in this study and compared to placebo. No CS safety issues were identified with the use of SkQ1 ophthalmic solution at either concentration. Rates of ocular and non-ocular AEs were similar between the SkQ1 treatment groups and the placebo group. No serious AEs (SAEs) occurred, and no subjects were discontinued due to AEs. A total of 18 treatment-emergent AEs (TEAEs) occurred in 
Table 3 Relationship of TEAEs to treatment

\begin{tabular}{|c|c|c|c|}
\hline \multirow{2}{*}{$\begin{array}{l}\text { Classification of TEAEs by } \\
\text { possible relationship to treatment }\end{array}$} & \multicolumn{3}{|c|}{ Reported TEAEs by treatment group } \\
\hline & SkQ1 $1.55 \mu \mathrm{g} / \mathrm{mL}$ & SkQ1 $0.155 \mu \mathrm{g} / \mathrm{mL}$ & Placebo \\
\hline \multicolumn{4}{|l|}{ Ocular } \\
\hline Suspected & & 1 (ocular discomfort) & 2 (blurred vision) \\
\hline Not suspected & 1 & & 1 \\
\hline \multicolumn{4}{|l|}{ Non-ocular } \\
\hline Suspected & & & 1 (headache) \\
\hline Not suspected & 6 & 5 & 1 \\
\hline Total & 7 & 6 & 5 \\
\hline
\end{tabular}

Suspected TEAE was suspected of being related to the treatment, not suspected TEAE was suspected of not being related to the treatment, TEAEs treatment-emergent adverse events

this study: 7 TEAEs in the SkQ1 $1.55 \mu \mathrm{g} / \mathrm{mL}$ group, 6 TEAEs in the SkQ1 $0.155 \mu \mathrm{g} / \mathrm{mL}$ group, and 5 TEAEs in the placebo group; only three of these TEAEs were suspected of being related to treatment ( 2 TEAEs in the placebo group, and one TEAE in the SkQ1 $0.155 \mu \mathrm{g} / \mathrm{mL}$ group), as shown in Table 3.

No effects on visual acuity, IOP, slit lamp biomicroscopy tests, or dilated fundoscopy were observed. Any abnormal findings were reported at baseline and all were considered not CS. Regarding visual acuity, there were no CS differences in the group mean visual acuity ( $\log$ MAR) scores between either of the SkQ1 treatment groups and the placebo group at any study visit. In addition, there were no CS differences noted among the treatment groups in the proportions of subjects with normal, abnormal (NCS), and abnormal (CS) slit lamp findings; the same was true for dilated fundoscopy findings. For IOP, there were no CS differences in mean IOP values or change in IOP between either concentration of SkQ1 and placebo at the two time points measured (Visit 1 and Visit 4).

Both concentrations of SkQ1 were demonstrated to be safe and well tolerated ophthalmic products for use in dry eye subjects.

\section{DISCUSSION}

\section{Oxidative Stress}

Oxidative stress is involved in a variety of diseases, including cancer, Alzheimer's disease, Parkinson's disease, and cardiovascular, lung, and skin diseases, as well as ocular conditions including macular degeneration, uveitis, cataracts, corneal and ocular surface inflammation, and dry eye [6, 33]. Oxidative stress occurs with excessive levels of ROS, a type of free radical produced as a byproduct of cellular mitochondrial respiration. At normal levels, ROS is important in cell signaling and homeostasis [6]. ROS, oxidative stress, and inflammation are important factors in dry eye disease, for which inflammation is considered the primary mechanism [31]. The relationship between oxidative stress and inflammation in dry eye disease continues to be explored. High levels of ROS and oxidative stress markers have been identified in the tear film and conjunctiva of Sjogren syndrome patients [32], in superficial punctate keratopathy in corneal epithelia [17], in the tear film of dry eye patients [1], and in animal models of dry eye [6]. 
Wakamatsu et al. [33] discussed antioxidant enzyme activity in the eye, with the highest levels found in the retina, lower levels in the sclera and cornea, and tears containing little such enzymatic activity [4]. Thus, except for the retina, the eye contains few protections against free radicals/ROS. It has been suggested that lactoferrin [24] or selenium [8] may provide protection of the corneal epithelium. SkQ1 belongs to a relatively new class of chemical compounds-mitochondria-targeted

antioxidants-capable of targeting and neutralizing mitochondrial ROS [10, 27].

\section{Aging, Environmental, and Lifestyle Contributors to Dry Eye Disease}

Aging, which commonly results in decreased tear production and flow, and certain hormonal changes (e.g., during menopause) are major contributors to dry eye disease, with oxidative stress involved in the natural aging process [33]. In addition, acute and chronic environmental conditions can contribute to oxidative stress in the ocular surface, resulting in dry eye. Environmental and lifestyle contributors to dry eye are numerous and include: exposure to low humidity outdoors or indoors; tobacco smoke; wind; sun [ultraviolet (UV)] exposure; pollutants; ozone; certain medications, such as antihistamines, antidepressants, and beta-blockers; certain medical conditions, including other ocular conditions (e.g., blepharitis, allergic conjunctivitis), rheumatoid arthritis, Sjogren's syndrome, or contact dermatitis; contact lens use; and activities that decrease blinking, such as prolonged computer use or reading. SKQ1 has been shown to play a key role in the treatment of age-related diseases $[11,16,28]$, slow age-dependent retinal degeneration [19], and increase the resistance of the lens to UV-irradiation [30].

\section{Dry Eye Models}

Animal and human models of dry eye can reflect the environmental conditions that contribute to oxidative stress and dry eye. A rat model of dry eye using low-humidity airflow suggested a strong relationship between oxidative stress and corneal surface disorder [17]. A mouse model reflecting age-related ocular changes in the retina indicated that oxidative stress may play a role in retinal degeneration, and that the superoxide dismutase (SOD) enzyme may protect retinal pigment epithelium from age-related degeneration [9]. Using this same mouse model, another study found that SOD1 may also provide protection for the dry eye parameters of tear function, the ocular surface, and the lacrimal gland [34].

SkQ1 or related molecules have been shown to be active in a variety of animal models of illness believed to involve free radical damage. These include rat models of $\mathrm{H}_{2} \mathrm{O}_{2}$ and ischemia-induced heart arrhythmia, heart infarction, kidney ischemia, and stroke, studied both ex vivo and in vivo [2]. In addition, SkQ1 has shown efficacy in a number of eye disease models. Ocular preclinical studies with SkQ1 have included dry eye models in mice, and uveitis and retinal degeneration models in rats $[14,20,30]$. In the Phase 2 US clinical trial, SkQ1 was investigated using the CAE model to reflect the oxidative stress conditions of dry eye. The post-CAE results of this study as reported here indicate that SkQ1 treatment prior to CAE exposure protects the ocular surface from oxidative stress and provides a novel approach to dry eye treatment.

\section{Preclinical Studies with SkQ1}

Preclinical studies have shown that SKQ1 effectively modulates mitochondria membrane 
electric potential, reduces cellular destruction and damage caused by excessive concentrations of ROS, and decreases ocular surface inflammation $[25,38]$. Based on a full range of animal toxicology studies, no adverse effects on general behavior or the central nervous system were observed (in rats orally administered SkQ1 at $0,5,25$, or $100 \mathrm{mg} / \mathrm{kg}$ ), or for cardiovascular parameters, pulse pressure, electrocardiography, body temperature, or clinical condition (in Beagle dogs orally administered SkQ1 at 0.00, $7.75,77.5$, or $775 \mu \mathrm{g} / \mathrm{kg}$ ) (unpublished reports). A mouse model study showed the efficacy of SkQ1 for the reduction of corneal staining, as discussed in the "Dose Selection" section above.

\section{Possible Study Limitations}

Limitations of some of the available diagnostic tools (e.g., Schirmer's test, corneal fluorescein staining) may contribute to mixed underlying etiologies. Use of several dry eye tests compensates somewhat for these test limitations. In addition, seasonality may contribute to a reduction in the effects of dry eye treatments as the drier season progresses. In addition, environmental factors, such as subjects' increased use of computers or reading or exposure to pollutants or certain medications, may also decrease the effects of dry eye treatments. Using a dry eye model (e.g., CAE chamber) that regulates humidity, temperature, airflow, lighting, and visual tasking, as used in the US Phase 2 study, can help control and minimize these potential confounding factors.

\section{Prior SkQ1 Clinical Studies}

The Phase 2 US clinical trial followed two clinical studies conducted in Russia. The first Russian clinical trial of SkQ1 demonstrated efficacy in treating signs and symptoms of dry eye compared to an artificial tear treatment [36], after which Visomitin SkQ1 ophthalmic solution was approved in Russia (December 2011) and has since been marketed there as a prescription product. The second Russian clinical study assessed the safety, tolerability, and efficacy of Visomitin compared with placebo (the same artificial tears formulation used in the initial Visomitin study) [5]. The results of the second Russian study indicated that SkQ1 was safe and well tolerated by subjects, and restored corneal cell function and tear film stability, with improvements in both symptoms and corneal staining associated with dry eye. Post-therapy efficacy of Visomitin for TBUT, corneal staining, and symptoms over 6 weeks of a follow-up observation period showed a clear advantage of Visomitin over artificial tear treatments. The US Phase 2 clinical trial confirmed the safety and efficacy of SkQ1 dry eye treatment.

For future studies, categorizing subjects by both their worst dry eye symptom score and their corneal fluorescein staining scores may be useful in determining treatment efficacy, particularly when changes from pre-CAE and post-CAE scores at the end of the four-week treatment cycle are compared to baseline pre- to post-CAE scores.

\section{CONCLUSIONS}

This Phase 2 clinical trial indicated that SkQ1 is efficacious for the treatment of dry eye signs and symptoms and supported prior study results. SkQ1 was safe for use in the study's dry eye subjects, who found SkQ1 to be well tolerated and comfortable. Future studies will be conducted to confirm the efficacy and safety of SkQ1 ophthalmic solution. 


\section{ACKNOWLEDGMENTS}

Sponsorship, article processing charges, and the open access charge for this study were funded by Mitotech S.A., Luxembourg. All authors had full access to all of the data in this study and take complete responsibility for the integrity of the data and accuracy of the data analysis.

All named authors meet the International Committee of Medical Journal Editors (ICMJE) criteria for authorship for this manuscript, take responsibility for the integrity of the work as a whole, and have given final approval for the version to be published.

Disclosures. A. Petrov is an employee of Mitotech S.A. N. Perekhvatova is an employee of Mitotech S.A. M. Skulachev is an employee of Mitotech S.A. and Mitotech LLC. L. Stein is an employee of Ora, Inc. G. Ousler is an employee of Ora, Inc.

Compliance with ethics guidelines. The study was Institutional review board-approved (Alpha IRB) and registered (Clinicaltrials.gov identifier: NCT02121301).

All procedures followed were in accordance with the ethical standards of the responsible committee on human experimentation (institutional and national) and with the Helsinki Declaration of 1964, as revised in 2013. Informed consent was obtained from all patients at the beginning of the study.

Open Access. This article is distributed under the terms of the Creative Commons Attribution-NonCommercial 4.0 International License (http://creativecommons.org/licenses/ by-nc/4.0/), which permits any noncommercial use, distribution, and reproduction in any medium, provided you give appropriate credit to the original author(s) and the source, provide a link to the Creative Commons license, and indicate if changes were made.

\section{REFERENCES}

1. Augustin AJ, Spitznas M, Kaviani N, Meller D, Koch $\mathrm{FH}$, Grus F, et al. Oxidative reactions in the tear fluid of patients suffering from dry eyes. Graefes Atch Clin Exp Ophthalmol. 1995;233(11):694-8.

2. Bakeeva LE, Barskov IV, Egorov MV, et al. Mitochondria-targeted plastoquinone derivatives as tools to interrupt execution of the aging program. 2. Treatment of some ROS- and age-related diseases (heart arrhythmia, heart infarctions, kidney ischemia, and stroke). Biochem Biokhimiia. 2008;73(12):1288-99.

3. Baudouin C. The pathology of dry eye. Surv Ophthalmol. 2001;45(Suppl. 2):S211-20.

4. Behndig A, Svensson B, Marklund SL, Karlsson K. Superoxide dismutase isoenzymes in the human eye. Invest Ophthalmol Vis Sci. 1998;39(3):471-5.

5. Brzheskiy VV, Alekseev VN, Gusarevich OG, et al. Results of a multicenter, randomized, double-masked, placebo-controlled clinical study of the efficacy and safety of Visomitin eye drops in patients with dry eye syndrome. Adv Ther. 2015;32:1263-79. doi:10.1007/s12325-0150273-6

6. Deng R, Hua X, Li J, Chi W, Zhang Z, Lu F, Zhang L, Pflugfelder S, Li D-Q. Oxidative stress markers induced by hyperosmolarity in primary human corneal epithelial cells. PLoS One. 2015;10(5): e0126561. doi:10.1371/journal.pone0126561.

7. Dry Eye Workshop (DEWS) Committee. 2007 report of the international dry eye workshop (DEWS. Ocular Surf. 2007;5(2):65-204.

8. Higuchi $A$, Inoue $H$, Kawakita $T$, Ogishima $T$, Tsubota K. Selenium compound protects corneal epithelium against oxidative stress. PLoS One. 2012;7(9):e45612. doi:10.1371/journal.pone. 0045612 .

9. Imamura $\mathrm{Y}$, Noda $\mathrm{S}$, Hashizume $\mathrm{K}$, Shinoda $\mathrm{K}$, Yamaguchi M, Uchiyama S, et al. Drusen, choroidal neovascularization, and retinal pigment epithelium dysfunction in SOD1-deficient mice: a model of age-related macular degeneration. Proc Natl Acad Sci. 2006;103(30):11282-7. 
10. Kelso G, Porteous C, Coulter C, Hughes G, Porteous W, Ledgerwood E, Smith R, Murphy M. Selective targeting of a redox-active ubiquinone to mitochondria within cells: antioxidant and antiapoptotic properties. J Biol Chem. 2001;276: 4588-96.

11. Kolosova NG, Stefanova NA, Muraleva NA, Skulachev VP. The mitochondria-targeted antioxidant SkQ1 but not $\mathrm{N}$-acetylcysteine reverses aging-related biomarkers in rats. Aging. 2012;4(10):686-94.

12. Korshunov SS, Skulachev VP, Starkov AA. High protonic potential actuates a mechanism of production of reactive oxygen species in mitochondria. FEBS Lett. 1997;416:15-8.

13. Lemp MA. Report of the National Eye Institute/ industry workshop on Clinical Trials in Dry Eye. CLAO J. 1995;21:221-32.

14. Markovets AM, Fursova AZ, Kolosova NG. Therapeutic action of the mitochondriatargeted antioxidant SkQ1 on retinopathy in OXYS rats linked with improvement of VEGF and PEDF gene expression. PLoS One. 2011;6(7):e21682.

15. Messmer E. The pathophysiology, diagnosis, and treatment of dry eye disease. Dtsch Arztebl Int. $2015 ; 112: 71-82$.

16. Muraleva NA, Kozhevnikova OS, Zhdankina AA, Stefanova NA, Karamysheva TV, Fursova AZ, et al. The mitochondria-targeted antioxidant SkQ1 restores alphaB-crystallin expression and protects against AMD-like retinopathy in OXYS rats. Cell cycle (Georgetown, Tex). 2014;13(22): 3499-505.

17. Nakamura S, Shibuya M, Nakashima H, Hisamura R, Masuda N, Imagawa $\mathrm{T}$, et al. Involvement of oxidative stress on corneal epithelial alterations in a blink-suppressed dry eye. IOVS. 2007;48(4): 1552-8.

18. Ousler GW, Gomes PJ, Welch D, Abelson MB. Methodologies for the study of ocular surface disease. Ocular Surf. 2005;3(3):143-54.

19. Saprunova VB, Lelekova MA, Kolosova NG, Bakeeva LE. SkQ1 slows development of age-dependent destructive processes in retina and vascular layer of eyes of wistar and OXYS rats. Biochem Biokhimiia. 2012;77(6):648-58.

20. Saprunova V, Pilipenko D, Alexeevsky A, Fursova A, Kolosova N, Bakeeva L. Lipofuscin granule dynamics during development of age-related macular degeneration. Biochem Biokhimiia. 2010;75(2):130-8.
21. Schaumberg D, Dana R, Buring J, Sullivan D. Prevalence of dry eye disease among US men: estimates from the Physicians' Health Studies. Arch Ophthalmol. 2009;127(6):763-8.

22. Schaumberg D, Sullivan D, Buring J, Dana M. Prevalence of dry eye syndrome among US women. Am J Ophtahlmol. 2003;136(2):318-26.

23. Sheppard J, Torkildsen G, Lonsdale J, D'Ambrosio F, McLaurin E, Eiferman R, Kennedy K, Semba C. Lifitegrast ophthalmic solution $5.0 \%$ for treatment of dry eye disease. Ophthalmology. 2014;121(2):475-83.

24. Shimmura S, Suematsu M, Shimoyama M, Tsubota K, Oguchi Y, Ishimura Y. Subthreshold UV radiation-induced peroxide formation in cultured corneal epithelial cells: the protective effects of lactoferrin. Exp Eye Res. 1996;63(5):519-26.

25. Skulachev VP. Cationic antioxidants as a powerful tool against mitochondrial oxidative stress. Biochem Biophys Res Commun. 2013;441(2):275-9.

26. Skulachev VP. What is "phenoptosis" and how to fight it? Biochem Biokhimiia. 2012;77(7):689-706.

27. Skulachev M, Antonenko Y, Anisimov V, Chernyak B, Cherepanov D, Chistyakov V, Egorov $M$, Kolosova N, Korshunova G, Lyamzaev K, Plotnikov E, Roginsky V, Savchenko A, Severina II, Severin F, Shkurat T, Tashlitsky V, Shidlovsky K, Vyssokikh M, Zamyatnin A Jr, Zorov D, Skulachev V. Mitochondrial-targeted plastoquinone derivatives. Effect on senescence and acute age-related pathologies. Curr Drug Targets. 2011;12:800-26.

28. Skulachev VP, Anisimov VN, Antonenko YN, Bakeeva LE, Chernyak BV, Erichev VP, et al. An attempt to prevent senescence: a mitochondrial approach. Biochim Biophys Acta. 2009;1787(5):437-61.

29. Skulachev VP. Functions of mitochondria: from intracellular power stations to mediators of a senescence program. Cell Mol Life Sci. 2009;66(11-12):1785-93.

30. Snytnikova O, Tsentalovich Y, Stefanova N, Fursova A, Kaptein R, Sagdeev R, et al. The therapeutic effect of mitochondria-targeted antioxidant SkQ1 and Cistanche deserticola is associated with increased levels of tryptophan and kynurenine in the rat lens. Dokl Biochem Biophys. 2012;447:300-3.

31. Stevenson W, Chauhan SK, Dana R. Dry eye disease: an immune-mediated ocular surface disorder. Arch Ophthalmol. 2012;130(1):90-100.

32. Wakamatsu TH, Dogru M, Matsumoto Y, Kojima T, Kaido M, Ibrahim OM, et al. Evaluation of lipid oxidative stress status in Sjogren syndrome patients. IOVS. 2013;54:201-10. 
33. Wakamatsu TH, Dogru M, Tsubota K. Tearful relations: oxidative stress, inflammation and eye diseases. Arq Bras Oftalmol. 2008;71(6 Suppl):72-9.

34. Wakamatsu T, Dogru M, Sasaki Y, Ward S, Imamura $\mathrm{Y}$. Histopathological alterations in senescent $\mathrm{Cu}$, Zn-superoxide dismutase-1 (Sod-1)-knock-out mice: a new model for dry eye. In: 5th international conference on the tear film and ocular surface, Taormina, Italy, 4-9 September 2007.

35. Whitlock A, Belen L, Violette K, Brackett J, Ousler G, Perekhvatova N. Evaluation of topical SKQ1 in a murine $\mathrm{CAE}^{\mathrm{TM}}$ model of dry eye disease. Abstract/poster presentation at The international symposium on ocular pharmacology and therapeutics 10th ISOPT clinical symposium, Paris, France, 7-10 March 2013.
36. Yani EV, Katargina LA, Chesnokova NB, Beznos OV, Savchenko AYU, Vygodin VA, Gudkova EYU, Zamyatnin AA, Skulachev MV. The first experience of using the drug Vizomitin in the treatment of dry eyes. Pract Med (Russia). 2012;4(59):134-7.

37. Yeh S, Song XJ, Farley W, et al. Apoptosis of ocular surface cells in experimentally induced dry eye. Invest Ophthalmol Vis Sci. 2003;44:124-9.

38. Zinovkin RA, Romaschenko VP, Galkin II, Zakharova VV, Pletjushkina OY, Chernyak BV, Popova EN. Role of mitochondrial reactive oxygen species in age-related inflammatory activation of endothelium. Aging. 2014;6(8):661-74. 